\title{
Fibroblast growth factor-23 may serve as a novel biomarker for renal osteodystrophy progression
}

\author{
SI YAN LIU, DONG DONG ZHANG, YANG FANG WU, HUANG HUANG LUO, \\ GUANG MEI JIANG, YAO XU, YUE WU, XUN XIA, WEI WEI, BO HU and PENG HU
}

Department of Pediatrics, The First Affiliated Hospital of Anhui Medical University, Hefei, Anhui 230022, P.R. China

Received February 12, 2018; Accepted October 9, 2018

DOI: 10.3892/ijmm.2018.3934

\begin{abstract}
The purpose of the present study was to determine whether fibroblast growth factor (FGF)-23 could serve as a novel biomarker for renal osteodystrophy (ROD) progression. A rat model of ROD was induced by left nephrectomy plus intravenous injection of Adriamycin. Serum FGF-23 was determined using an enzyme-linked immunosorbent assay. Serum level and bone expression of FGF-23 were both significantly elevated in the ROD group at $24 \mathrm{~h}$ post-surgery. Serum FGF-23 was negatively correlated with calcium, phosphate, 25-hydroxyvitamin $\mathrm{D}$, conventional bone biomarkers and bone collagen X. More importantly, serum FGF-23 was significantly associated with abnormalities in bone formation rate, osteoblasts, osteoclasts, trabecular volume thickness and osteoid volume. Therefore, FGF-23 may serve as a novel biomarker for ROD.
\end{abstract}

\section{Introduction}

Renal osteodystrophy (ROD), a complex bone disorder, mainly results from hormonal dysmetabolism during the progression

Correspondence to: Professor Peng Hu, Department of Pediatrics, The First Affiliated Hospital of Anhui Medical University, $218 \mathrm{Ji}-\mathrm{Xi}$ Road, Hefei, Anhui 230022, P.R. China

E-mail: hupeng28@aliyun.com

Abbreviations: ABD, adynamic bone disease; Alb, Serum albumin; bAP, bone-specific alkaline phosphatase; BUN, blood urea nitrogen; CKD, chronic kidney disease; Col X, collagen X; DPD, urinary deoxypyridinoline; FGF-23, fibroblast growth factor-23; FGFR, fibroblast growth factor receptor; ICTP, cross-linked carboxyterminal telopeptide of type I collagen; OC, osteocalcin; PICP, procollagen type I carboxy-terminal propeptide; PTH, parathyroid hormone; PYD, pyridinoline; ROD, renal osteodystrophy; SCr, serum creatinine; SHPT, secondary hyperparathyrodism; tAP, total alkaline phosphatase; TRAP, artrate-resistant acid phosphatase; UA, uric acid; UCr, urine creatinine; UP, urinary protein; XLH, X-linked hypophosphatemic

Key words: Adriamycin-induced nephropathy, collagen X, fibroblast growth factor-23, parathyroid hormone, renal osteodystrophy of chronic kidney disease (CKD). In 1983, Sherrard et al (1) recommended a classification system for ROD using the parameters of bone turnover and the unmineralized bone (osteoid) area as a percentage of total bone area and fibrosis. On the basis of these criteria, ROD can be classified into three main categories: Osteitis fibrosa characterized by high bone turnover, adynamic bone disease (ABD) with low bone turnover, and mixed lesion (2). In general, osteitis fibrosa does not cause mineralization abnormalities, but stimulates secondary hyperparathyrodism (SHPT) and eventually the excessive proliferation of osteocytes in long bones. A cross-sectional study conducted by Mathias et al (3) recruited 21 patients with osteitis fibrosa, and found that total alkaline phosphatase (tAP) and parathyroid hormone (PTH) were markedly increased whereas the serum calcium level was decreased. Conversely, $\mathrm{ABD}$ is characterized by the mineralization abnormality of bone collagen and absence of osteoid accumulation in combination with relative hypoparathyroidism. Moore et al (4) determined serum biomarkers of bone and mineral metabolism in 43 African-American hemodialysis patients and revealed that ABD exhibited significantly decreased levels of serum phosphorus, calcium-phosphorus product, PTH, tAP and bone-specific alkaline phosphatase (bAP). Nevertheless, the diagnostic efficiency of routine serum biomarkers is far from satisfactory. Ferreira et al (5) discovered that an elevation in PTH (>300 pg/ml) together with higher tAP (>120 U/l) had a low sensitivity $(58.8 \%)$ and negative predictive value $(44.0 \%)$ for the diagnosis of osteitis fibrosa. Similarly, another study comprising 93 CKD patients from 18 Macedonian ambulatory nephrology centers indicated that the sensitivity of PTH $(\leq 237 \mathrm{pg} / \mathrm{ml})$ for ABD diagnosis was $78 \%$, and specificity was 53\%; the sensitivity and specificity of tAP ( $\leq 66 \mathrm{U} / \mathrm{l})$ were 74 and 54\%, respectively (6). Although bone biopsy is considered the gold standard for ROD diagnosis, the invasiveness seriously restricts its clinical application. Therefore, further studies are warranted to screen novel biomarkers that should be unique to mineral metabolism and be well correlated with histomorphometric findings in ROD progression.

Fibroblast growth factor (FGF)-23, originally identified in human osteoblasts, is an important hormonal regulator of circulating phosphate and calcitriol via suppressing the $\mathrm{Na} / \mathrm{Pi}$ IIc cotransporters in proximal tubule cells (7). In the concurrent presence of FGF receptor (FGFR)-1 and the transmembrane protein Klotho, FGF-23 also antagonizes $1 \alpha$-hydroxylase and 
subsequently leads to 1,25-dihydroxy vitamin D deficiency and calcium malabsorption, which may initiate the development of bone and mineral disorders (8). Bai et al (9) generated transgenic mice overexpressing human FGF-23 and revealed that FGF-23 transgenic mice recapitulated the biochemical (hypophosphatemia, hypovitaminosis D and decreased renal tubular phosphate reabsorption) and skeletal abnormalities (rickets and osteomalacia) consistent with human X-linked hypophosphatemic (XLH) rickets and autosomal dominant hypophosphatemic rickets. More directly, Wang et al (10) transfected human FGF-23 into osteoblasts in vitro and found that overexpressed FGF-23 could significantly disturb osteoblast differentiation and matrix mineralization. In addition, a growing body of literature has noted that elevated FGF-23 is a common feature in CKD. In an animal study conducted by Parker et al (11), serum FGF-23 was significantly higher in CKD dogs compared with in control dogs (467 vs. $315 \mathrm{pg} / \mathrm{ml}$ ). In agreement with the aforementioned report, a prospective study including 3,879 CKD patients showed that serum FGF-23 was $236 \mathrm{RU} / \mathrm{ml}$ (IQR 160-372 RU/ml) in patients with end-stage kidney disease, a value 5.5 -fold higher than baseline values, $43 \mathrm{RU} / \mathrm{ml}$ (12). However, there is scant information currently available on the involvement of FGF-23 in ROD progression to date.

\section{Materials and methods}

Animals and treatment. Male Sprague-Dawley rats $(\mathrm{n}=96)$ weighing 120-150 $\mathrm{g}$ had access to standard diet and water and were housed with constant temperature $\left(23 \pm 1^{\circ} \mathrm{C}\right)$ and humidity $(55 \pm 5 \%)$, and a 12-h light/dark cycle. All animal experimentation was in accordance with the guidelines of the Institutional Animal Use and Care Committee, Anhui Medical University. According to the protocol of Kim et al (13), rats were grouped using a block randomization method, and divided equally between the ROD and sham-operated (SO) groups. Then, 48 rats in each group were randomly divided into 8 subgroups using the same method. Randomization sequences were created using Excel 2007 (Microsoft Corporation, Redmond, WA, USA) with a 1:1 allocation using random block sizes of 8 . Rats were operated on under anesthesia with intraperitoneal injections of pentobarbital $(60 \mathrm{mg} / \mathrm{kg}$ body weight). The ROD model was induced with 48 rats via left nephrectomy plus intravenous injection of Adriamycin $(5 \mathrm{mg} / \mathrm{kg}$ body weight; Shenzhen Wanle Pharmaceutical Co. Ltd., Shenzhen, China), dissolved in $0.9 \%$ saline. The other 48 rats formed the SO group and underwent a sham laparotomy with ureteric manipulation through a midline incision. Subsequently, animals in both groups were anesthetized by intraperitoneal pentobarbital injection and sacrificed by cardiac puncture at $24 \mathrm{~h}, 72 \mathrm{~h}$, 1 week, 2 weeks, 3 weeks, 1 month, 2 months or 3 months after surgery (depending on subgroup). According to the disease duration, the 8 subgroups were classified into acute stage (disease duration $\leq 1$ week), advanced stage ( 1 week $<$ disease duration $\leq 1$ month), or chronic stage ( 1 month $<$ disease duration $\leq 3$ months).

Laboratory analysis. All rats were individually housed in metabolic cages and urine was collected for $24 \mathrm{~h}$. Urine creatinine concentration (UCr) and urinary protein concentration (UP) from the sample at $24 \mathrm{~h}$ were determined using the biuret colorimetric method (Boehringer Mannheim, Milan, Italy). Blood samples were obtained from the abdominal aorta and centrifuged at $1,600 \mathrm{x}$ g for $15 \mathrm{~min}$ and $4^{\circ} \mathrm{C}$. Serum albumin (Alb), blood urea nitrogen (BUN), serum creatinine ( $\mathrm{SCr}$ ), uric acid (UA), calcium and phosphate were measured by standard enzymatic methods (Randox Laboratories Ltd., Crumlin, UK). The serum protein levels of FGF-23, 25-hydroxyvitamin D [25-(OH)D], parathyroid hormone (PTH), osteocalcin (OC), bone-specific alkaline phosphatase (bAP), total alkaline phosphatase (tAP), procollagen type I carboxy-terminal propeptide (PICP), cross-linked carboxyterminal telopeptide of type I collagen (ICTP), tartrate-resistant acid phosphatase (TRAP), urinary deoxypyridinoline (DPD) and pyridinoline (PYD) were determined using commercially available enzyme-linked immunosorbent assay (ELISA) kits (Invitrogen; Thermo Fisher Scientific, Inc.), according to the manufacturer's protocols.

Kidney histology. Once harvested, the kidney was washed with saline and blotted on gauze. Kidney sections were fixed in $4 \%$ paraformaldehyde and embedded in paraffin. Paraffin-embedded sections ( $4-\mu \mathrm{m}$ thick) were stained with hematoxylin and eosin. Renal morphologic lesions were evaluated on ten randomly selected, non-overlapping specimens from each rat at $x 400$ magnification, and examined independently by two pathologists blinded to the experimental design.

Bone morphology. At harvest, the distal femurs were excised, placed in $70 \%$ ethanol, and dehydrated in increasing concentrations of ethanol. Specimens were embedded in methyl methacrylate and not decalcified. Bones were sectioned $(4-\mu \mathrm{m})$ longitudinally through the frontal plane using a microtome (Leica, Wetzlar, Germany), and stained with Goldner's trichrome for histomorphological analysis.

Reverse transcription (RT)-PCR. Total RNA was isolated from bone tissues using TRIzol ${ }^{\circledR}$ reagent (Invitrogen; Thermo Fisher Scientific, Inc.), using a routine protocol. Ultraviolet spectrophotometry to measure absorbance, and agarose gel electrophoresis, confirmed no RNA degradation. cDNA was synthesized from $1 \mu \mathrm{g}$ total RNA using an ExScript RT reagent kit (Takara Biotechnology, Dalian, China).

Primers for FGF-23, FGFR-1, Klotho, Collagen (Col)-X and GAPDH are listed in Table I. RT-PCR was performed with an AB detection system and SYBR Green ${ }^{\circledR}$ PCR Master Mix (Applied Biosystems; Thermo Fisher Scientific, Inc.) in accordance with the manufacturer's instructions. Amplification conditions were as follows: Pre-denaturing at $95^{\circ} \mathrm{C}$ for $2 \mathrm{~min}$; denaturing at $94^{\circ} \mathrm{C}$ for $20 \mathrm{sec}$, annealing at $60^{\circ} \mathrm{C}$ for $20 \mathrm{sec}$, extension at $72^{\circ} \mathrm{C}$ for $30 \mathrm{sec}$; and, finally, one cycle of $5 \mathrm{~min}$ at $72^{\circ} \mathrm{C}$. GAPDH was also measured in each sample and used as an internal control for loading and reverse transcription efficiency. The average threshold cycle $(\mathrm{Ct}$, the cycles of template amplification to the threshold) was calculated for each sample. The quantitative $2^{-\Delta \Delta \mathrm{Ct}}$ was used to compare relative mRNA expression $(14,15)$.

Western blot analysis. Bone tissues were homogenized in ice-cold buffer (40 mM KCl, $10 \mathrm{mM}$ HEPES, $\mathrm{pH} 7.9$, 
Table I. Primers used in RT-PCR.

\begin{tabular}{lll}
\hline Genes & \multicolumn{1}{c}{ Forward (5'-3') } & \multicolumn{1}{c}{ Reverse $\left(5^{\prime}-3^{\prime}\right)$} \\
\hline FGF-23 & GATGCTGGCTCCGTAGTGATAAT & TGATGCTTCGGTGACAGGTAGA \\
FGFR-1 & GGCACCTGAGGCATTGTTT & TACTGGGCTTGTCCATTCG \\
Klotho & GCCGAGCAAGACTCACTG & GCAAAGTAGCCACAAAGGT \\
Col X & TCTGGGATGCCTCTTGTC & GATCTTGGGTCATAGTGCTG \\
GAPDH & GTTACCAGGGCTGCCTTCTC & GGGTTTCCCGTTGATGACC
\end{tabular}

$3 \mathrm{mM} \mathrm{MgCl}{ }_{2}, 5 \%$ glycerol, $0.5 \mu \mathrm{g} / \mathrm{ml}$ leupeptin, $0.1 \mu \mathrm{g} / \mathrm{ml}$ aprotinin, $1.5 \mu \mathrm{g} / \mathrm{ml}$ pepstatin, and $100 \mathrm{mg} / \mathrm{ml}$ phenylmethylsulphonyl fluoride) with a Polytron homogenizer for 15-20 sec. Homogenates were centrifuged at $500 \mathrm{x} \mathrm{g}$ for $10 \mathrm{~min}$ at $41^{\circ} \mathrm{C}$, following which supernatants were re-centrifuged at $12,000 \times \mathrm{g}$ for $15 \mathrm{~min}$ at $41^{\circ} \mathrm{C}$. The pellets were resuspended in $0.5 \mathrm{ml}$ homogenizing buffer containing $0.5 \%$ Nonidet P-40 (Wuhan Boster Biological Technology, Ltd., Wuhan, China) and the total protein concentration of each supernatant was determined by the dye binding method using bovine serum albumin as the standard. Samples $(50 \mu \mathrm{g})$ were subjected to $10 \%$ SDS-PAGE and transferred onto a nitrocellulose membrane (Bio-Rad Laboratories, Inc.- Hercules, CA, USA). The membranes were sealed with Tris-buffered saline and $0.1 \%(\mathrm{w} / \mathrm{v})$ Tween-20 (TBST) containing 5\% non-fat dry milk (Bio-Rad Laboratories, Inc.) overnight. Immunoblots were incubated with anti-FGF-23, Klotho, FGFR-1, Col X and GAPDH primary antibodies (Medical Discovery Leader Biotech Co., Ltd., Beijing, China; www.Mdlbiotech.com) at $4^{\circ} \mathrm{C}$ overnight (Table II). The membranes were washed three times (10 min each) with TBST, followed by incubation with horseradish peroxidase-conjugated anti-rabbit immunoglobulin $\mathrm{G}$ antibodies (1:3,000 dilution; Medical Discovery Leader) at room temperature $\left(25^{\circ} \mathrm{C}\right)$ for $60 \mathrm{~min}$. The reaction products were assessed using an enhanced chemiluminescence detection system and exposed to radiographic film for variable periods.

Statistical analysis. All data are expressed as the means \pm SEM. The Student's two-sample t-test was used for comparisons of normally distributed variables with equal variances between two groups. Comparisons of mean values among multiple groups were conducted using one-way ANOVA and the Student-Newman-Keuls post hoc test. A value of $\mathrm{P}<0.05$ was considered to indicate a statistically significant difference. Pearson's correlation coefficients (r) were reported for serum FGF-23 and biochemical parameters (calcium, phosphate, 25-(OH)D, OC, tAP, bAP, PICP, ICTP, TRAP, PYD, DPD and Col X). Pearson's correlation coefficient ( $r$ ) is a measure of the linear correlation between two variables $X$ and $Y$. It has a value between +1 and -1 , where 1 is total positive linear correlation, 0 is no linear correlation, and -1 is total negative linear correlation. Statistical analyses were performed using the SPSS v.17.0 software (SPSS, Inc., Chicago, IL, USA).

\section{Results}

Renalfunction indices. Renal function indices of the ROD and SO groups are listed in Table III. Compared with 24 h post-surgery,
UA in the ROD group was significantly increased at $72 \mathrm{~h}$ and 3 months post-surgery; SCr and Ucr were significantly higher at the advanced and chronic stages, whereas BUN was decreased at the advanced and chronic stages $(\mathrm{P}<0.01)$. Compared with the SO group, BUN and UA in the ROD group were significantly higher in each stage, $\mathrm{SCr}$ and UCr were notably elevated at the advanced and chronic stages, and UPr markedly increased only at 1 -month post-surgery $(\mathrm{P}<0.05)$. Nevertheless, Alb in the ROD group was significantly lower compared with the SO group, throughout the observational period $(\mathrm{P}<0.05)$.

Serum FGF-23. Serum level of FGF-23 is presented in Fig. 1A. Compared with 24 h post-surgery, serum FGF-23 in the ROD group was significantly elevated at 2 months and 3 months post-surgery $(\mathrm{P}<0.05)$. Compared with the SO group, serum FGF-23 was significantly higher in the ROD group at all the time-points $(\mathrm{P}<0.05)$. Furthermore, the highest level of serum FGF-23 appeared at 3 months $(164.37 \mathrm{pg} / \mathrm{ml})$, which is $\sim 3.29$-fold higher than the SO group baseline level (49.95 $4.25 \mathrm{pg} / \mathrm{ml})$.

Bone metabolism indices. Changes in serum calcium, phosphate, 25-(OH)D, PTH, osteoblastic and osteoclastic proteins are shown in Fig. 1. Compared with at $24 \mathrm{~h}$ post-surgery, PTH was significantly elevated in the ROD group at 3 weeks post-surgery $(\mathrm{P}<0.01)$, though no significant differences in calcium, phosphate and $25-(\mathrm{OH}) \mathrm{D}$ were observed among the time-points $(\mathrm{P}>0.05)$. At the time of sacrifice, the levels of calcium, phosphate and $25-(\mathrm{OH}) \mathrm{D}$ were significantly decreased in the ROD group in every stage compared with in the respective SO group $(\mathrm{P}<0.05)$ (Fig. 1B-D). By contrast, serum PTH was significantly higher than in the SO group, from the acute to the chronic stage $(\mathrm{P}<0.05)$ (Fig. 1E). Osteoblastic $(\mathrm{OC}$, PICP, tAP and bAP) (Fig. 1F-I) and osteolytic proteins (ICTP, TRAP, PYD and DPD) (Fig. 1J-M) were notably different between the two groups, and the ROD group expressed significantly lower levels of bone turnover markers relative to the SO group during the present study $(\mathrm{P}<0.05)$. Compared with at $24 \mathrm{~h}$ post-surgery, tAP, TRAP and ICTP in the ROD group were significantly increased at 1 week, 1 week and 3 weeks post-surgery, respectively $(\mathrm{P}<0.05)$.

Kidney histology. Histological images of the kidney are shown in Fig. 2. By contrast with the SO group, renal injury in the ROD group gradually deteriorated over time. Renal damage was limited to epithelial cell swelling, accompanied by inflammatory cell infiltration in both the glomerulus and interstitium during the acute and advanced stages; 

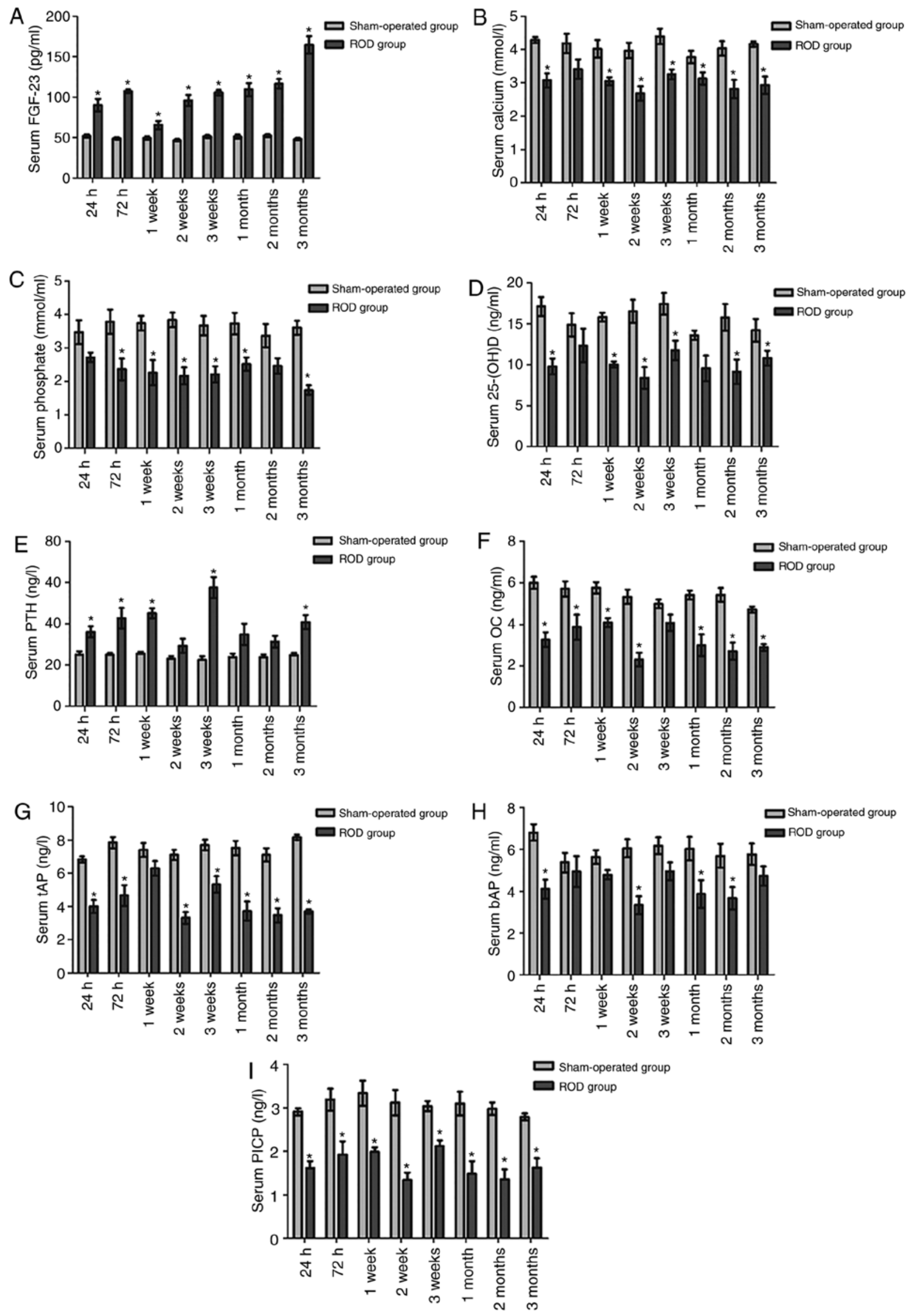

Figure 1. Serum FGF-23, calcium, phosphate, 25-(OH)D, PTH, osteoblastic and osteoclastic proteins in ROD and SO rats. (A) Serum FGF-23 was significantly higher in the ROD group at all the time-points ( $\mathrm{P}<0.05)$. (B-D) The levels of calcium, phosphate and 25-(OH)D were significantly decreased in the ROD group at every stage compared with the SO group $(\mathrm{P}<0.05)$. (E) Serum PTH was significantly higher than that in the SO group, from the acute stage to the chronic stage $(\mathrm{P}<0.05)$. (F-I) Osteoblastic proteins (OC, PICP, tAP and bAP) were significantly lower, relative to the SO group $(\mathrm{P}<0.05)$. 

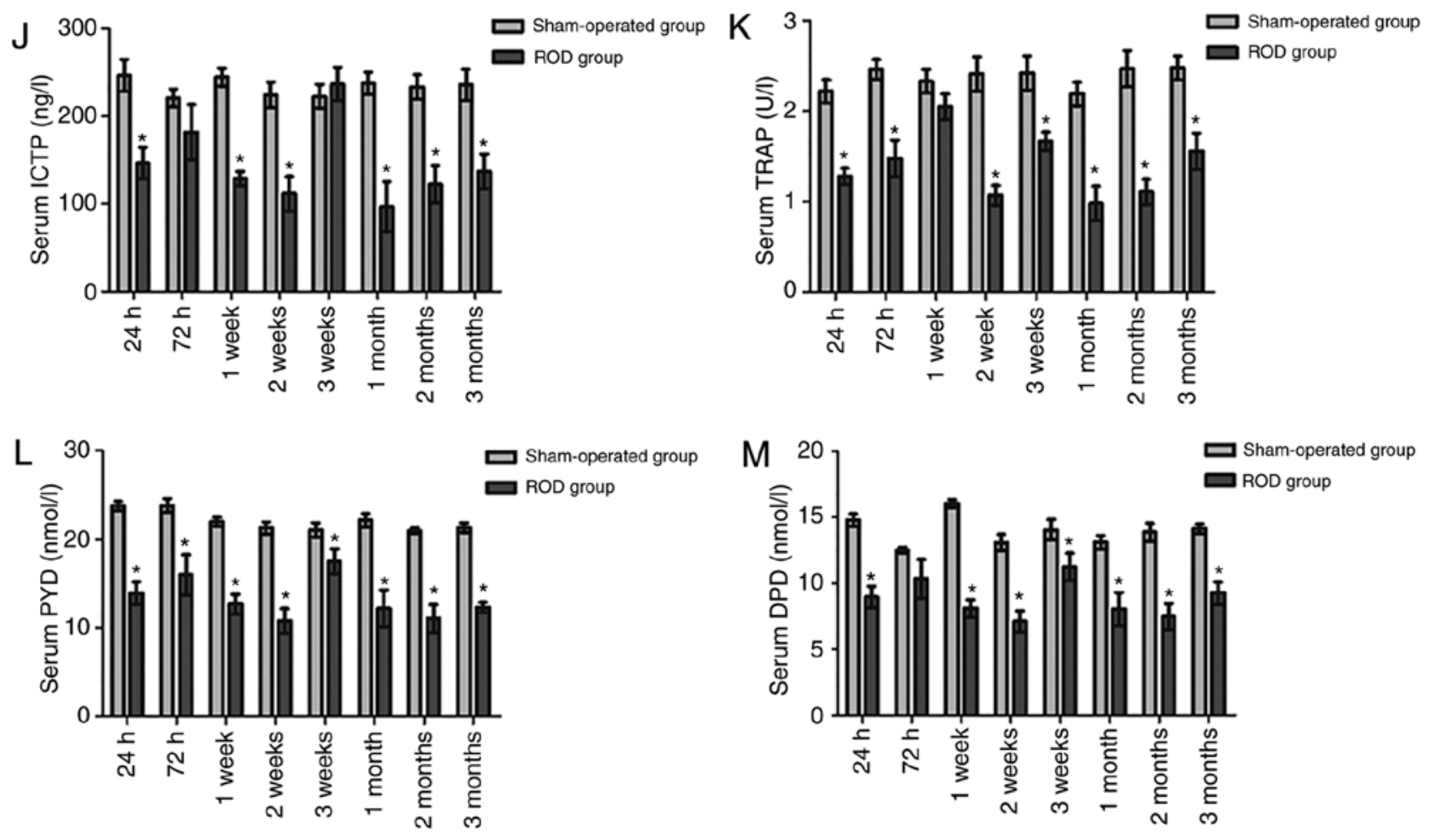

Figure 1. Continued. (J-M) Osteolytic proteins (ICTP, TRAP, PYD and DPD) were significantly lower, relative to the SO group (P<0.05). ${ }^{*} \mathrm{P}<0.05$, significantly different from the corresponding SO group. ROD, renal osteodystrophy; SO, sham operated; 25-(OH)D, 25-hydroxyvitamin D; bAP, bone-specific alkaline phosphatase; DPD, deoxypyridinoline; FGF-23, fibroblast growth factor-23; ICTP, cross-linked carboxyterminal telopeptide of type I collagen; OC, osteocalcin; PICP, procollagen type I carboxy-terminal propeptide; PTH, parathyroid hormone; PYD, urinary pyridinoline; tAP, total alkaline phosphatase; TRAP, tartrate-resistant acid phosphatase.

when progressing to the chronic stage, renal pathology was characterized by inflammatory cell infiltration and tubule collapse. By contrast, kidney sections from the SO group had a normal appearance during the whole observational period.

Bone morphology. Representative histological images of distal femurs are shown in Fig. 3. In the acute stage, there was no notable difference in bone histology between the ROD and SO groups. Pathological lesions in the ROD group occurred at the advanced stage and were gradually aggravated thereafter. Compared with the SO group, Adriamycin induced a low-turnover bone lesion characterized by a marked decrease in bone formation rate, osteoblasts, osteoclasts, and trabecular volume thickness, and a clear elevation in osteoid volume.

Bone expression of FGF-23, Klotho, FGFR-1 and Col X. Bone expression levels of FGF-23, Klotho, FGFR-1 and Col X at the time of sacrifice in the ROD and SO groups are shown in Fig. 4. Compared with at $24 \mathrm{~h}$ post-surgery, FGF-23 and FGFR-1 mRNA levels in the ROD group were significantly increased at the chronic stage; Klotho mRNA significantly increased at $72 \mathrm{~h}, 1,2$ and 3 months post-surgery, and Col X mRNA were significantly elevated at 3 months post-surgery $(\mathrm{P}<0.05)$. The ROD group had significant increases in FGF-23 and Klotho mRNA expression compared with the SO group at each stage $(\mathrm{P}<0.05)$ (Fig. 4A and B), and increased FGFR-1 mRNA at the advanced and chronic stages $(\mathrm{P}<0.05)$ (Fig. 4C). In the ROD group, the highest expression levels for FGF-23 mRNA were at 3 months (10.63-fold), and the highest expression levels for Klotho and FGFR-1 mRNA were at 2 months (3.83- and 6.24-fold, respectively).

On the contrary, Col X mRNA in the ROD group was significantly reduced, with a $94 \%$ decrease at $72 \mathrm{~h}$ $(\mathrm{P}<0.05)$ (Fig. 4D). Subsequently, western blotting was conducted to evaluate the bone expression levels of FGF-23, FGFR-1, Klotho and Col X protein (Fig. 4I). Compared with at $24 \mathrm{~h}$ post-surgery, FGF-23, Klotho and FGFR-1 protein in the ROD group were significantly higher at $72 \mathrm{~h}, 2$ months and 3 months post-surgery; and $\mathrm{Col} \mathrm{X}$ were significantly upregulated at the advanced and chronic stages $(\mathrm{P}<0.05)$. The ROD group exhibited incremental values of FGF-23 and FGFR-1 protein expression, as compared with the SO group, at each stage $(\mathrm{P}<0.05)$ (Fig. 4E and $\mathrm{G})$, whereas a significantly higher Klotho level was observed only in the acute and chronic stages $(\mathrm{P}<0.05)$ (Fig. 4F). In the ROD group, the highest expression for FGF-23 and FGFR-1 proteins was at $72 \mathrm{~h}$ (6.63- and 1.46-fold, respectively). Furthermore, the highest expression levels of Klotho were exhibited at 3 months (1.27-fold). By contrast, the expression of Col X protein was significantly suppressed in ROD rats throughout the observational period and showed the largest downregulation (57\% decrease) at 2 weeks $(\mathrm{P}<0.05)$ (Fig. $4 \mathrm{H})$.

Associations between serum FGF-23 levels and biochemical parameters. Associations between serum FGF-23 levels and biochemical parameters in ROD rats are depicted in Fig. 5. Serum FGF-23 was negatively correlated with calcium $(r=-0.585, \mathrm{P}<0.01$, Fig. 5A), phosphate $(r=-0.645, \mathrm{P}<0.01$, 
Fig. 5B), 25-(OH)D ( $r=-0.532$, P $<0.01$, Fig. 5C), OC $(r=-0.607$, $\mathrm{P}<0.01$, Fig. 5E), tAP $(r=-0.718, \mathrm{P}<0.01$, Fig. 5F), bAP $(r=-0.503, \mathrm{P}<0.01$, Fig. 5G), PICP $(r=-0.696, \mathrm{P}<0.01$, Fig. 5H), ICTP $(r=-0.572, \mathrm{P}<0.01$, Fig. 5I), TRAP $(r=-0.699, \mathrm{P}<0.01$, Fig. 5J), PYD ( $r=-0.505, \mathrm{P}<0.01$, Fig. 5K), DPD $(r=-0.232$, $P=0.023$, Fig. 5L $)$ and Col $\mathrm{X}(r=-0.244, \mathrm{P}=0.017$, Fig. $5 \mathrm{~N})$. As expected, serum FGF-23 was positively correlated with bone expression levels of the FGF-23 protein $(r=+0.772, \mathrm{P}<0.01$, Fig. 5M) and serum PTH ( $r=+0.399, \mathrm{P}<0.01$, Fig. 5D).

\section{Discussion}

Adriamycin-induced nephropathy is often recognized as a classical model for gaining valuable insights into the molecular and cellular mechanisms of CKD and its complications $(16,17)$. However, it remains to be identified whether Adriamycin triggers bone and mineral disorders on the basis of nephrotoxicity. In 1980, Van Vleet and Ferrans (18) first studied chronic Adriamycin $(2.4 \mathrm{mg} / \mathrm{kg} / \mathrm{w})$ intoxication in 50 weanling rabbits for $\leq 17$ weeks; prominent renal damage was selective for the inner cortex, and other lesions contained skeletal muscle degeneration and osteodystrophy-associated fractures. Coincidentally, 2 decades later, Ishii et al (19) established a uremic model in Sprague-Dawley rats by a single peritoneal injection of $5 \mathrm{mg} / \mathrm{kg}$ Adriamycin, and found that the glomerular filtration rate (GFR) and serum 25-(OH)D were reduced by 52 and $77 \%$, respectively, whereas PTH had a 2.5 -fold increase after 14 weeks. The Adriamycin-injected rats could also develop a low-turnover ROD resembling osteomalacia, as evidenced by a 4.3 -fold increase in osteoid volume, a $73 \%$ reduction in BFR, a 56\% decrease in adjusted apposition rate, and a 5-fold increase in mineralization lag time. As predicted, the present study confirmed the nephrotoxicity of Adriamycin, indicated by significantly increased BUN, $\mathrm{SCr}, \mathrm{UA}, \mathrm{UCr}, \mathrm{UPr}$ and decreased serum Alb. Progressive loss of renal function is one of the major manifestations of Adriamycin toxicity in animals. Wu et al (20) found that Adriamycin-injected mice developed renal dysfunction characterized by elevated BUN and SCr at day 28. Similarly, our study also revealed that both BUN and $\mathrm{SCr}$ increased markedly in the ROD group at the advanced stage, which may be due to Adriamycin-induced reduction of glomerular size, mesangial expansion, tubular atrophy, tubulointerstitial fibrosis and glomerular sclerosis (21). GFR is considered the best overall index for the assessment of renal function, usually assessed by two main routes: First, measuring the urinary excretion rate of a tracer using timed urine collections (22); second, determination of the plasma elimination kinetics of a tracer after a single bolus injection (23). However, urine collection is difficult to conduct precisely, and rats cannot tolerate repeated blood draws. Therefore, GFR was not measured in this study. In addition, Adriamycin injection could result in significant reductions of serum calcium, phosphate, 25-(OH) $\mathrm{D}$ and bone turnover markers, except for PTH. Furthermore, bone morphology demonstrated low-turnover lesions associated with Adriamycin in the present study.

FGF-23 is a bone-derived hormone that negatively regulates serum phosphate and bone formation $(24,25)$. A number of recent publications based on clinical and experimental research suggest that serum FGF-23 is elevated 
Table III. Renal function indices in ROD and SO rats.

\begin{tabular}{|c|c|c|c|c|c|c|}
\hline Categories & Alb (g/l) & BUN (mmol/l) & $\mathrm{SCr}(\mathrm{smol} / \mathrm{l})$ & $\mathrm{UA}(\mu \mathrm{mol} / \mathrm{l})$ & $\mathrm{UCr}(\mu \mathrm{mol} / \mathrm{l})$ & UPr (mg) \\
\hline \multicolumn{7}{|l|}{ SO group } \\
\hline $24 \mathrm{~h}$ & $34.83 \pm 2.36$ & $7.02 \pm 1.18$ & $17.83 \pm 9.58$ & $64.25 \pm 7.55$ & $32.40 \pm 9.26$ & $2.90 \pm 0.85$ \\
\hline $72 \mathrm{~h}$ & $35.60 \pm 2.03$ & $7.06 \pm 1.41$ & $18.09 \pm 3.78$ & $62.24 \pm 3.14$ & $31.49 \pm 5.32$ & $3.08 \pm 0.77$ \\
\hline 1 week & $33.52 \pm 2.05$ & $6.80 \pm 1.40$ & $18.73 \pm 6.94$ & $60.72 \pm 3.33$ & $32.06 \pm 4.36$ & $3.17 \pm 0.80$ \\
\hline 2 weeks & $34.15 \pm 1.71$ & $6.88 \pm 1.74$ & $21.61 \pm 5.44$ & $60.06 \pm 1.14$ & $30.33 \pm 5.19$ & $3.25 \pm 0.44$ \\
\hline 3 weeks & $34.86 \pm 1.75$ & $6.12 \pm 1.02$ & $19.53 \pm 5.43$ & $61.00 \pm 3.65$ & $32.06 \pm 5.01$ & $2.90 \pm 0.87$ \\
\hline 1 month & $33.65 \pm 2.13$ & $7.22 \pm 1.48$ & $19.50 \pm 5.38$ & $61.29 \pm 2.24$ & $31.15 \pm 6.91$ & $3.62 \pm 0.80$ \\
\hline 2 months & $34.38 \pm 2.65$ & $5.87 \pm 1.38$ & $17.86 \pm 6.82$ & $61.12 \pm 2.60$ & $31.42 \pm 5.93$ & $3.36 \pm 0.93$ \\
\hline 3 months & $35.28 \pm 1.93$ & $6.93 \pm 0.80$ & $20.40 \pm 9.41$ & $59.83 \pm 5.91$ & $31.60 \pm 9.26$ & $3.18 \pm 1.42$ \\
\hline \multicolumn{7}{|l|}{ ROD group } \\
\hline $24 \mathrm{~h}$ & $30.98 \pm 2.06^{\mathrm{a}}$ & $14.66 \pm 5.57^{\mathrm{a}}$ & $15.50 \pm 3.39$ & $52.00 \pm 13.58$ & $17.50 \pm 6.60^{\mathrm{a}}$ & $4.41 \pm 1.52$ \\
\hline $72 \mathrm{~h}$ & $32.38 \pm 2.03^{\mathrm{a}}$ & $13.31 \pm 3.83^{\mathrm{a}}$ & $17.20 \pm 4.17$ & $95.21 \pm 26.16^{\mathrm{a}, \mathrm{b}}$ & $50.00 \pm 58.23$ & $2.88 \pm 1.11$ \\
\hline 1 week & $33.00 \pm 3.88$ & $8.15 \pm 0.93^{\mathrm{b}}$ & $20.00 \pm 3.41$ & $54.83 \pm 16.92$ & $52.00 \pm 34.85^{\mathrm{b}}$ & $4.50 \pm 2.08$ \\
\hline 2 weeks & $31.49 \pm 2.01^{\mathrm{a}}$ & $8.48 \pm 1.04^{b}$ & $27.67 \pm 3.50^{\mathrm{a}, \mathrm{b}}$ & $70.80 \pm 15.07$ & $56.60 \pm 36.63^{b}$ & $4.54 \pm 2.18$ \\
\hline 3 weeks & $27.80 \pm 5.70^{a}$ & $9.58 \pm 1.15^{\mathrm{a}}$ & $19.67 \pm 2.34$ & $75.00 \pm 8.69 a$ & $71.67 \pm 38.95^{\mathrm{b}}$ & $5.52 \pm 3.00$ \\
\hline 1 month & $30.77 \pm 1.19^{a}$ & $10.19 \pm 1.13^{\mathrm{a}}$ & $27.00 \pm 3.03^{\mathrm{a}, \mathrm{b}}$ & $79.17 \pm 34.08$ & $276.80 \pm 157.37^{\mathrm{a}, \mathrm{b}}$ & $22.14 \pm 12.85^{\mathrm{a}, \mathrm{b}}$ \\
\hline 2 months & $34.28 \pm 2.87^{\mathrm{b}}$ & $9.24 \pm 1.74^{\mathrm{a}, \mathrm{b}}$ & $33.50 \pm 4.80^{\mathrm{a}, \mathrm{b}}$ & $79.00 \pm 2.61^{\mathrm{a}, \mathrm{b}}$ & $1,094.60 \pm 661.09^{\mathrm{a}, \mathrm{b}}$ & $11.67 \pm 8.40$ \\
\hline 3 months & $29.24 \pm 3.58^{\mathrm{a}}$ & $11.04 \pm 3.87^{\mathrm{a}}$ & $33.75 \pm 3.37^{\mathrm{a}, \mathrm{b}}$ & $87.00 \pm 8.22^{\mathrm{a}, \mathrm{b}}$ & $1,632.33 \pm 491.43^{\mathrm{a}, \mathrm{b}}$ & $5.39 \pm 3.74$ \\
\hline
\end{tabular}

${ }^{a} \mathrm{P}<0.05$, significantly different from the corresponding SO group. ${ }^{\mathrm{b}} \mathrm{P}<0.05$, significantly different from rats at $24 \mathrm{~h}$ post-operation.

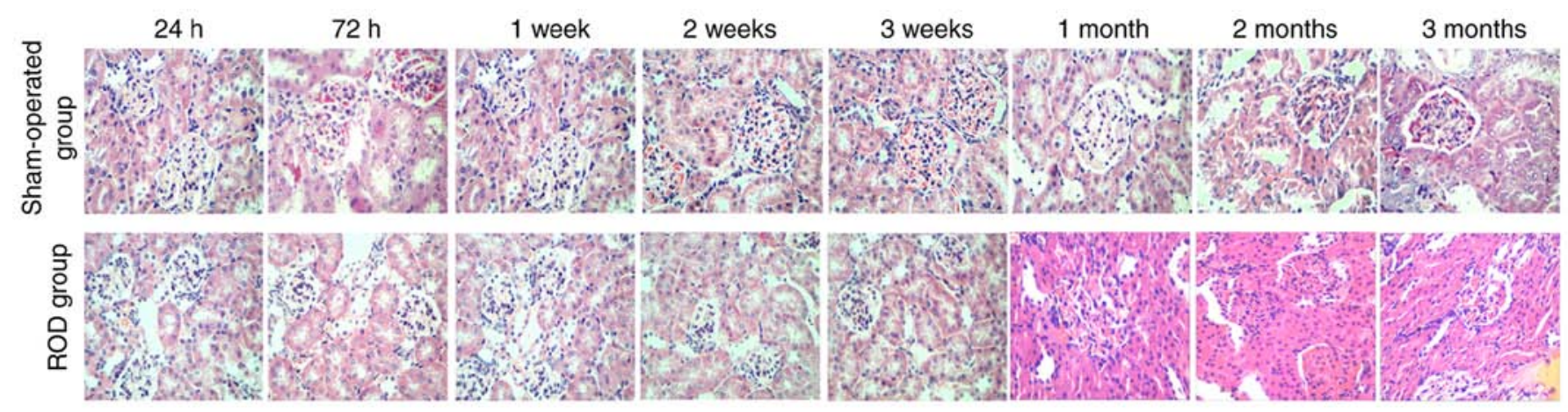

Figure 2. Representative histological images of kidneys in ROD and SO rats. Different from the SO group, renal injury of the ROD group gradually deteriorated over time. Renal damage was limited to epithelial cell swelling, accompanied by inflammatory cell infiltration in both the glomerulus and interstitium during the acute and advanced stage; when progressing to the chronic stage, renal pathology was characterized by inflammatory cell infiltration and tubule collapse. By contrast, kidney sections of the SO group had a normal appearance during the whole observational period. ROD, renal osteodystrophy; SO, sham operated.

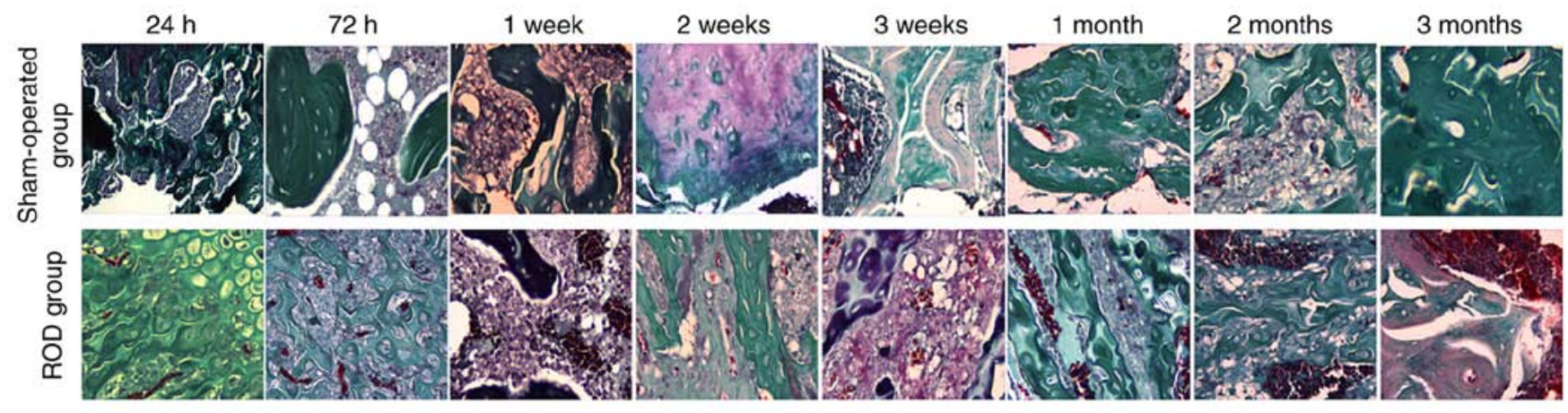

Figure 3. Representative histological images of bone in ROD and SO rats. In the acute stage, there was no notable difference in bone histology between the two groups. Pathological lesions of the ROD group occurred at the advanced stage and gradually aggravated thereafter. Characterized by a low-turnover bone lesion characterized by a marked decrease in bone formation rate, osteoblast, osteoclast and trabecular volume thickness, and an obvious elevation in osteoid volume. ROD, renal osteodystrophy; SO, sham operated. 

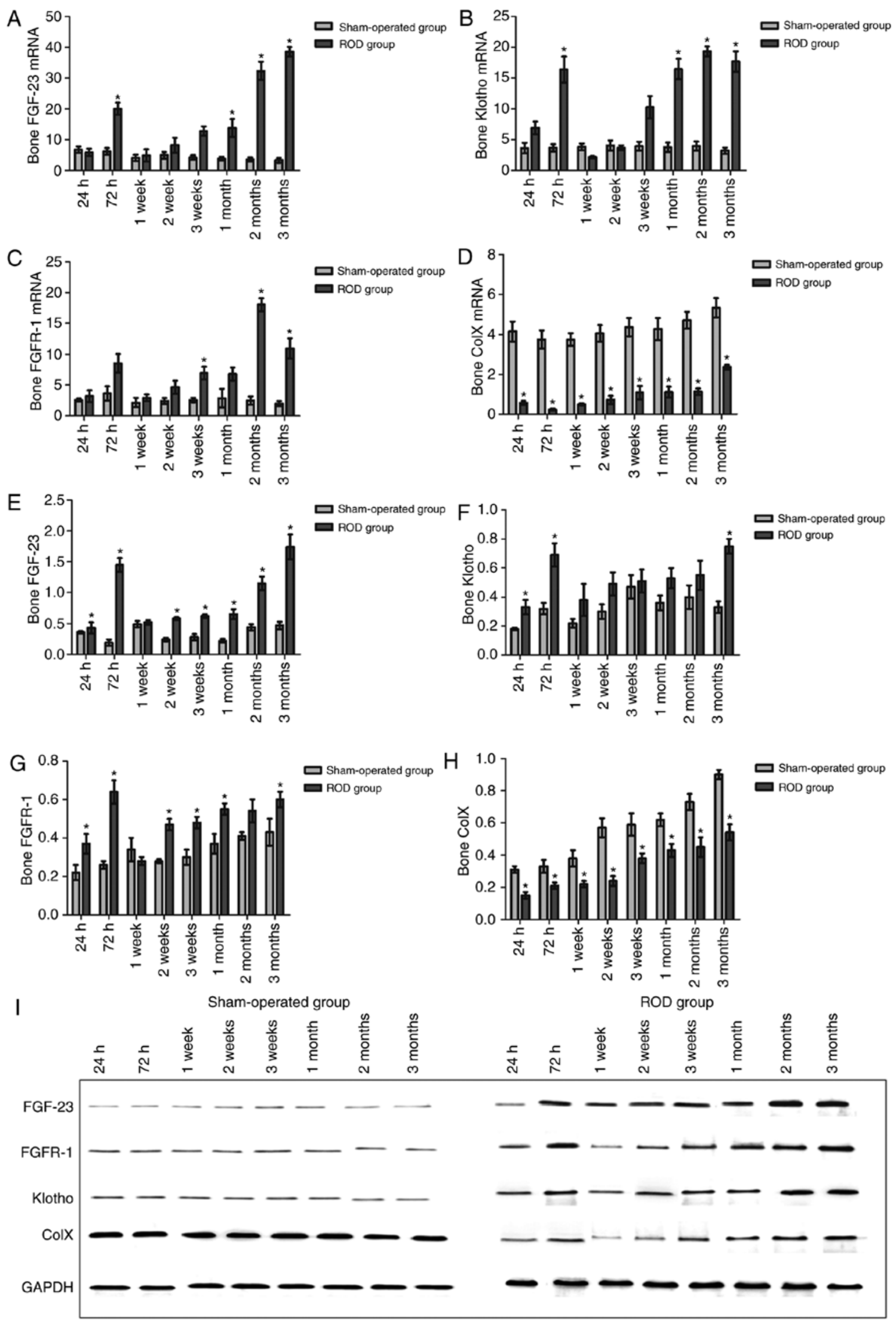

Figure 4. The bone expressions of FGF-23, FGFR-1, Klotho and Col X in ROD and SO rats. (A and B) The ROD group had significant elevations in FGF-23 and Klotho mRNA expression compared with the SO group in each stage $(\mathrm{P}<0.05)$, and $(\mathrm{C})$ increased FGFR-1 mRNA at the advanced and chronic stages $(\mathrm{P}<0.05)$. (D) On the contrary, Col X mRNA in the ROD group was significantly decreased at each time-point $(\mathrm{P}<0.05)$. (E and G) The ROD group exhibited incremental increases in FGF-23 and FGFR-1 protein as compared with the SO group at each stage ( $<<0.05)$, $(\mathrm{F})$ whereas a significantly higher Klotho level was observed only in the acute stage and chronic stage $(\mathrm{P}<0.05)$. $(\mathrm{H})$ By contrast, the expression of $\mathrm{Col} \mathrm{X}$ protein was significantly suppressed in the ROD rats throughout the observational period $(\mathrm{P}<0.05)$. (I) Representative western blots showing total protein expressions of bone FGF-23, FGFR-1, Klotho and Col X in ROD and SO rats. "P<0.05, significantly different from the corresponding SO group. ROD, renal osteodystrophy; SO, sham operated; Col X, collagen X; FGF-23, fibroblast growth factor-23; FGFR-1, fibroblast growth factor receptor-1. 

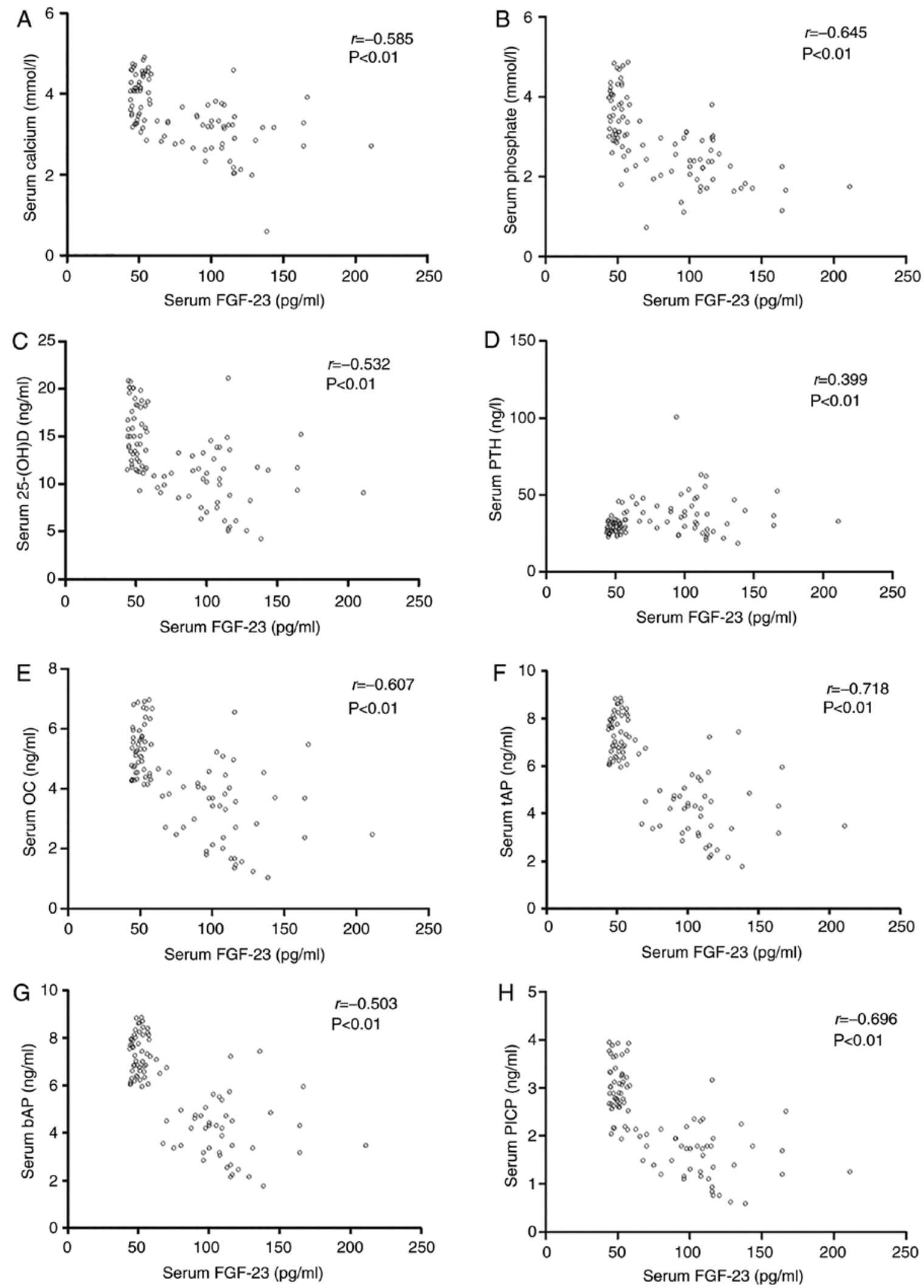

Figure 5. Associations between serum FGF-23 levels and biochemical parameters in ROD rats. Serum FGF-23 was negatively correlated with (A) calcium, (B) phosphate, (C) 25-(OH)D, (E) OC, (F) tAP, (G) bAP, (H) PICP. However, serum FGF-23 levels were positively correlated with bone expression levels of (D) serum PTH.

in CKD. A randomized controlled trial enrolling 556 children with CKD from 55 centers documented that the average serum FGF-23 was $183 \mathrm{kRU} / 1$ among all patients and inversely correlated with GFR; specifically, it gradually increased with the disease duration (128 kRU/l in stage 3,226 kRU/l in stage 4, and $654 \mathrm{kRU} / 1$ in stage 5) (26). In an animal study conducted by Hasegawa et al (27), progressive nephritis was induced in Wistar-Kyoto rats by single injection of anti-glomerular 

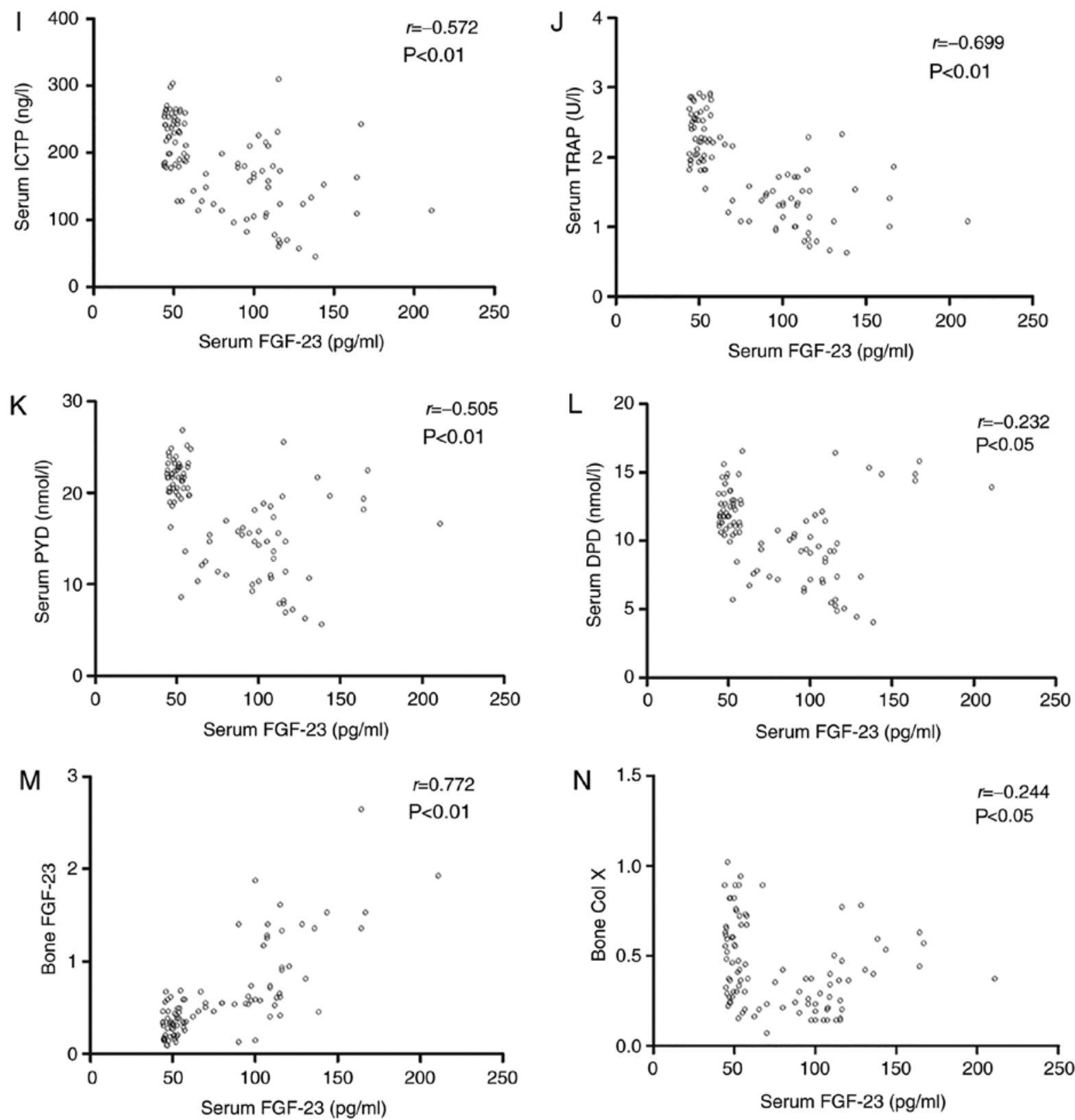

Figure 5. Continued. Serum FGF-23 was negatively correlated with (I) ICTP, (J) TRAP, (K) PYD, (L) DPD and (N) Col X. However, serum FGF-23 levels were positively correlated with bone expression levels of (M) FGF-23 protein. ROD, renal osteodystrophy; 25-(OH)D, 25-hydroxyvitamin D; bAP, bone-specific alkaline phosphatase; DPD, deoxypyridinoline; FGF-23, fibroblast growth factor-23; ICTP, cross-linked carboxyterminal telopeptide of type I collagen; OC, osteocalcin; PICP, procollagen type I carboxy-terminal propeptide; PTH, parathyroid hormone; PYD, urinary pyridinoline; tAP, total alkaline phosphatase; TRAP, tartrate-resistant acid phosphatase.

basement membrane (GBM) antiserum. Serum FGF-23 showed a moderate but significant increase by day 10 and rapidly increased thereafter in rats with anti-GBM nephritis. In the present study, serum FGF-23 was significantly higher in the Adriamycin-injected rats throughout the observational period, when compared with their counterparts, and the highest level appeared at 3 months. However, whether FGF-23 serves as a biomarker for ROD progression remains unknown.

FGF-23 cellular signaling requires the concurrent presence of FGFR-1, -2, -3, -4 and Klotho. Among the four receptors, FGFR-1 has the highest affinity for FGF-23 (28). The findings of this study revealed that elevated serum FGF-23 was accompanied by the overexpression of bone FGF-23, FGFR-1 and Klotho in the ROD group. Mace et al (29) established a
5/6 nephrectomy rat model to examine the regulation of FGF-23 expression, and found that serum and bone-derived FGF-23 levels were markedly upregulated in uremic rats at 8 weeks post-surgery; conversely, the influence of FGFR signaling was examined using the FGFR inhibitor PD173074, which resulted in not only the suppression of serum FGF-23, but also the downregulation of bone-derived FGF-23 in both uremic and normal control rats after 5-h of exposure. More directly, Shalhoub et al (30) studied the effects of exogenous FGF-23 on osteoblastic MC3T3.E1 cell proliferation and differentiation for 14 days in vitro and observed that FGF-23 plus Klotho produced significant inhibition of mineralization and osteoblast activity through upregulating FGFR-1; conversely, these effects could be fully blocked by the FGFR-1 inhibitor SU5402. 
Col $\mathrm{X}$ is encoded by the COL1OAl gene and synthesized by terminally differentiated chondrocytes. Currently, Col X is the only known specific molecular marker for new bone formation during endochondral ossification $(31,32)$. Chung et al (33) generated transgenic mice carrying a dominant interference mutation in Col X and observed the development of craniofacial skeletal abnormalities, including skull and mandibular dysplasia. In addition, genetic variation of COL10A1 has been verified as the molecular basis of human Schmid metaphyseal chondrodysplasia (SMCD). Chan et al (34) studied Col X mutations in the growth plate cartilage of patients with SMCD and discovered that a nonsense mutation in the carboxyl-terminal domain not only changed the $\operatorname{Tyr}^{632}$ codon (TAC) to a stop codon (TAA), but that it also caused Col X haploinsufficiency. In the present study, $\mathrm{Col} \mathrm{X}$ expression was significantly suppressed and negatively correlated with FGF-23 in ROD rats throughout the observational period. In a transgenic mouse model, Wang et al (35) documented a notable elevation in serum and bone FGF23 accompanied by downregulated Col X in the growth plates. By contrast, blocking FGF-23 action could reverse Col $\mathrm{X}$ downregulation and improve the skeletal phenotype in XLH mice (36).

The last objective of the present study was to determine whether serum FGF-23 could reflect changes in conventional mineral and bone biomarkers in ROD progression. Our study showed that serum FGF-23 was negatively correlated with calcium, phosphate, 25-(OH)D and bone turnover biomarkers. Similarly, Kanda et al (37) examined the association between serum FGF-23 and bone-metabolism-associated markers in patients with CKD and found that serum FGF-23 was negatively correlated with calcium and $1,25(\mathrm{OH})_{2} \mathrm{D}$. Furthermore, multivariate logistic regression analysis showed that FGF-23 was independently associated with vertebral fractures, and the optimal cutoff level of FGF-23 indicative of vertebral fracture was $56.8 \mathrm{pg} / \mathrm{ml}$ (sensitivity, 0.82; specificity, 0.63). Specifically, FGF-23 expression was negative for the markers of late osteogenic differentiation, bone sialoprotein and $\mathrm{OC}$ in human trabecular bone cells in vitro (38).

In conclusion, a biomarker for ROD should be non-invasive, detectable early on, and consistent with bone pathological changes. This study showed that serum level and bone expression of FGF-23 were both significantly elevated in the ROD group since $24 \mathrm{~h}$ post-surgery. Serum FGF-23 was significantly associated with abnormalities in bone formation rate, osteoblast, osteoclast, trabecular volume thickness, and osteoid volume. Therefore, FGF-23 may serve as a novel biomarker for ROD. However, the present study is still only observational and exploratory, and more evidence will be collated in the future. Even though information on this hypothesis is still scarce, in vitro studies have been initiated in our laboratory to verify the data.

\section{Acknowledgements}

Not applicable.

\section{Funding}

This study was supported by the National Natural Science Foundation of China (grant no. 81570637).

\section{Availability of data and materials}

The datasets generated during and/or analyzed during the current study are available from the corresponding author on reasonable request.

\section{Authors' contributions}

$\mathrm{PH}$ and $\mathrm{BH}$ conceived and supervised the study; SYL, DDZ, HHL, YFW, GMJ, YX and YW performed experiments; SYL and DDZ analyzed data; SYL and PH wrote the manuscript; $\mathrm{XX}$ and WW reviewed the manuscripts. All authors approved the final version of the manuscript.

\section{Ethics approval and consent to participate}

All animal experimentation was undertaken in accordance with the guidelines of the Institutional Animal Use and Care Committee, Anhui Medical University.

\section{Patient consent for publication}

Not applicable.

\section{Competing interests}

The authors declare no conflict of interest.

\section{References}

1. Sherrard DJ, Hercz G, Pei Y, Maloney NA, Greenwood C, Manuel A, Saiphoo C, Fenton SS and Segre GV: The spectrum of bone disease in end stage renal failure-an evolving disorder. Kidney Int 43: 436-442, 1993.

2. Afifi A: Renal osteodystrophy in developing countries. Artif Organs 26: 767-769, 2002.

3. Mathias R, Salusky I, Harman W, Paredes A, Emans J, Segre G and Goodman W: Renal bone disease in pediatric and young adult patients on hemodialysis in a children's hospital. J Am Soc Nephrol 3: 1938-1946, 1993.

4. Moore C, Yee J, Malluche H, Rao DS, Monier-Faugere MC, Adams E, Daramola-Ogunwuyi O, Fehmi H, Bhat $S$ and Osman-Malik Y: Association between bone histology and markers of bone and mineral metabolism in african-american hemodialysis patients. Clin J Am Soc Nephrol 4: 1484-1493, 2009.

5. Ferreira A, Saraiva M, Behets G, Macedo A, Galvão M, D'Haese P and Drüeke TB: Evaluation of bone remodeling in hemodialysis patients: Serum biochemistry, circulating cytokines and bone histomorphometry. J Nephrol 22: 783-793, 2009.

6. Bervoets AR, Spasovski GB, Behets GJ, Dams G, Polenakovic MH, Zafirovska K, Van Hoof VO, De Broe ME and D'Haese PC: Useful biochemical markers for diagnosing renal osteodystrophy in predialysis end-stage renal failure patients. Am J Kidney Dis 41: 997-1007, 2003.

7. Portale AA, Zhang MY, David V, Martin A, Jiao Y, Gu W and Perwad F: Characterization of FGF23-dependent Egr-1 cistrome in the mouse renal proximal tubule. PLoS One 10: e0142924, 2015.

8. Hu P, Xuan Q, Hu B, Lu L, Wang J and Qin YH: Fibroblast growth factor- 23 helps explain the biphasic cardiovascular effects of vitamin D in chronic kidney disease. Int J Biol Sci 8: 663-671, 2012.

9. Bai X, Miao D, Li J, Goltzman D and Karaplis AC: Transgenic mice overexpressing human fibroblast growth factor 23 (R176Q) delineate a putative role for parathyroid hormone in renal phosphate wasting disorders. Endocrinology 145: 5269-5279, 2004.

10. Wang H, Yoshiko Y, Yamamoto R, Minamizaki T, Kozai K, Tanne K, Aubin JE and Maeda N: Overexpression of fibroblast growth factor 23 suppresses osteoblast differentiation and matrix mineralization in vitro. J Bone Miner Res 23: 939-948, 2008. 
11. Parker VJ, Harjes LM, Dembek K, Young GS, Chew DJ and Toribio RE: Association of Vitamin D metabolites with parathyroid hormone, fibroblast growth factor-23, calcium, and phosphorus in dogs with various stages of chronic kidney disease. J Vet Intern Med 31: 791-798, 2017.

12. Isakova T, Xie H, Yang W, Xie D, Anderson AH, Scialla J, Wahl P, Gutiérrez OM, Steigerwalt S, He J, et al; Chronic renal Insufficiency Cohort (Cric) Study Group: Fibroblast growth factor 23 and risks of mortality and end-stage renal disease in patients with chronic kidney disease. JAMA 305: 2432-2439, 2011.

13. Kim J and Shin W: How to do random allocation (randomization). Clin Orthop Surg 6: 103-109, 2014.

14. Livak KJ and Schmittgen TD: Analysis of relative gene expression data using real-time quantitative PCR and the 2(-Delta Delta C(T)) method. Methods 25: 402-408, 2001.

15. Schmittgen TD and Livak KJ: Analyzing real-time PCR data by the comparative C(T) method. Nat Protoc 3: 1101-1108, 2008.

16. Hu P, Qin YH, Pei J, Lei FY, Hu B and Lu L: Beneficial effect of all-trans retinoic acid (ATRA) on glomerulosclerosis rats via the downregulation of alpha-smooth muscle actin: A comparative study between ATRA and benazepril. Exp Mol Pathol 89: 51-57, 2010 .

17. Okuda S, Oh Y, Tsuruda H, Onoyama K, Fujimi S and Fujishima M: Adriamycin-induced nephropathy as a model of chronic progressive glomerular disease. Kidney Int 29: 502-510, 1986.

18. Van Vleet JF and Ferrans VJ: Clinical and pathologic features of chronic adriamycin toxicosis in rabbits. Am J Vet Res 41: 1462-1469, 1980

19. Ishii H, Wada M, Furuya Y, Nagano N, Nemeth EF and Fox J: Daily intermittent decreases in serum levels of parathyroid hormone have an anabolic-like action on the bones of uremic rats with low-turnover bone and osteomalacia. Bone 26: 175-182, 2000.

20. Wu HJ, Yiu WH, Wong DW, Li RX, Chan LY, Leung JC, Zhang Y, Lian Q, Lai KN, Tse HF, et al: Human induced pluripotent stem cell-derived mesenchymal stem cells prevent adriamycin nephropathy in mice. Oncotarget 8: 103640-103656, 2017.

21. Wang Y, Wang YP, Tay YC and Harris DC: Progressive adriamycin nephropathy in mice: Sequence of histologic and immunohistochemical events. Kidney Int 58: 1797-1804, 2000.

22. Brod J and Sirota JH: The renal clearance of endogenous 'creatinine' in man. J Clin Invest 27: 645-654, 1948.

23. Cousins C, Mohammadtaghi S, Mubashar M, Strong R, Gunasekera RD, Myers MJ and Peters AM: Clearance kinetics of solutes used to measure glomerular filtration rate. Nucl Med Commun 20: 1047-1054, 1999.

24. Hu P, Huang BY, Xia X, Xuan Q, Hu B and Qin YH: Therapeutic effect of CNP on renal osteodystrophy by antagonizing the FGF-23/MAPK pathway. J Recept Signal Transduct Res 36: 213-219, 2016

25. Wolf M: Update on fibroblast growth factor 23 in chronic kidney disease. Kidney Int 82: 737-747, 2012.
26. Doyon A, Fischer DC, Bayazit AK, Canpolat N, Duzova A Sözeri B, Bacchetta J, Balat A, Büscher A,Candan C, et al: Markers of bone metabolism are affected by renal function and growth hormone therapy in children with chronic kidney disease. PLoS One 10: e0113482, 2015.

27. Hasegawa H, Nagano N, Urakawa I, Yamazaki Y, Iijima K, Fujita T, Yamashita T, Fukumoto S and Shimada T: Direct evidence for a causative role of FGF23 in the abnormal renal phosphate handling and vitamin D metabolism in rats with early-stage chronic kidney disease. Kidney Int 78: 975-980, 2010.

28. Urakawa I, Yamazaki Y, Shimada T, Iijima K, Hasegawa H, Okawa K, Fujita T, Fukumoto S and Yamashita T: Klotho converts canonical FGF receptor into a specific receptor for FGF23. Nature 444: 770-774, 2006.

29. Mace ML, Gravesen E, Nordholm A, Hofman-Bang J, Secher T, Olgaard K and Lewin E: Kidney fibroblast growth factor 23 does not contribute to elevation of its circulating levels in uremia. Kidney Int 92: 165-178, 2017.

30. Shalhoub V, Ward SC, Sun B, Stevens J, Renshaw L, Hawkins N and Richards WG: Fibroblast growth factor 23 (FGF23) and alpha-klotho stimulate osteoblastic MC3T3.E1 cell proliferation and inhibit mineralization. Calcif Tissue Int 89: 140-150, 2011.

31. Schmid TM and Linsenmayer TF: Immunohistochemical localization of short chain cartilage collagen (type X) in avian tissues. J Cell Biol 100: 598-605, 1985.

32. Kwan AP, Cummings CE, Chapman JA and Grant ME: Macromolecular organization of chicken type $\mathrm{X}$ collagen in vitro. J Cell Biol 114: 597-604, 1991.

33. Chung KS, Jacenko O, Boyle P, Olsen BR and Nishimura I: Craniofacial abnormalities in mice carrying a dominant interference mutation in type X collagen. Dev Dyn 208: 544-552, 1997.

34. Chan D, Weng YM, Graham HK, Sillence DO and Bateman JF: A nonsense mutation in the carboxyl-terminal domain of type $X$ collagen causes haploinsufficiency in schmid metaphyseal chondrodysplasia. J Clin Invest 101: 1490-1499, 1998.

35. Wang X, Wang S, Li C, Gao T, Liu Y, Rangiani A, Sun Y, Hao J, George A, Lu Y, et al: Inactivation of a novel FGF23 regulator, FAM20C, leads to hypophosphatemic rickets in mice. PLoS Genet 8: e1002708, 2012.

36. Liu ES, Martins JS, Raimann A, Chae BT, Brooks DJ, Jorgetti V, Bouxsein ML and Demay MB: 1,25-Dihydroxyvitamin D alone improves skeletal growth, microarchitecture, and strength in a murine model of XLH, despite enhanced FGF23 expression. J Bone Miner Res 31: 929-939, 2016.

37. Kanda E, Yoshida M and Sasaki S: Applicability of fibroblast growth factor 23 for evaluation of risk of vertebral fracture and chronic kidney disease-mineral bone disease in elderly chronic kidney disease patients. BMC Nephrol 13: 122, 2012.

38. Riminucci M, Collins MT, Fedarko NS, Cherman N, Corsi A, White KE, Waguespack S, Gupta A, Hannon T, Econs MJ, et al: FGF-23 in fibrous dysplasia of bone and its association to renal phosphate wasting. Clin J Invest 112: 683-692, 2003. 